\title{
Precision moulding of biomimetic disposable chips for droplet-based applications
}

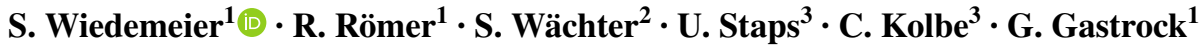

Received: 24 April 2017 / Accepted: 13 October 2017 / Published online: 25 October 2017

(C) The Author(s) 2017. This article is an open access publication

\begin{abstract}
In this study, we present a biomimetic approach to improve the stability and reproducibility of droplet generation processes and to reduce the adhesion of aqueous droplets to channel surfaces of microfluidic polymer chips. The hierarchical structure of the lotus leaf was used as a template for a partial laser structuring of the moulds that were used for casting the polymer chips. The hydrophobic wax layer of the lotus leaf was technologically replicated by coating the polymer chips using a plasma deposition process. The resulting microfluidic polymer chip surfaces reveal a topography and a surface free energy similar to those of the lotus leaf. Subsequent droplet-based microfluidic experiments were performed using a 2D flow focussing set-up. Droplets from
\end{abstract}

\section{S. Wiedemeier}

stefan.wiedemeier@iba-heiligenstadt.de

http://www.iba-heiligenstadt.de

R. Römer

http://www.iba-heiligenstadt.de

S. Wächter

http://www.ifw-jena.de

U. Staps

http://www.fkttriptis.de

C. Kolbe

http://www.fkttriptis.de

G. Gastrock

http://www.iba-heiligenstadt.de

1 Department for Bioprocess Engineering, Institute for Bioprocessing and Analytical Measurement Techniques e.V. (iba), Rosenhof, 37308 Heilbad Heiligenstadt, Germany

2 Department for Laser Technology, Guenter Koehler Institute for Joining Technology and Materials Testing GmbH (ifw), Otto-Schott-Str. 13, 07745 Jena, Germany

3 FKT Mold Construction and Plastics Technology GmbH, Jahnstraße 2, 07819 Triptis, Germany both, serum-supplemented cell culture medium and anticoagulated human whole blood, could be generated stably and reproducibly using a fluorocarbon as continuous phase. The presented results illustrate the application potential of the lotus-leaf-like polymer chips in life sciences, e.g. in the field of personalised medicine.

Keywords Droplet-based microfluidics · Injection moulding $\cdot$ Lotus effect $\cdot$ Surface structuring
Abbreviations
2D Two dimensional
3D Three dimensional
AFM Atomic force microscopy
DMEM Dulbecco's Modified Eagle's Medium
$\mathrm{C}_{4} \mathrm{~F}_{8} \quad$ Octafluorocyclobutane
$\mathrm{CV}$ Coefficient of variation
COC Cyclic olefin copolymer
FMS Fluid micro system
MST Test elements without microfluidic channels
OS Optical sensor
OWRK Owens, Wendt, Rabel and Kaelble method
pbb Pipe-based bioreactors
PC Polycarbonat
FEP Fluoroethylenpropylene
PFD Perfluorodecaline
PR Phenol red
PTFE Polytetrafluoroethylene
$Q_{\text {c }} \quad$ Volumetric flow rate of the continuous phase (organic phase, separation medium, perfluorodecaline)
$Q_{\mathrm{d}} \quad$ Volumetric flow rate of the disperse phase (aqueous or sample phase, biological media)
$s_{\mathrm{D}, \text { OS }} \quad$ Droplet length average calculated with the opti- cal detector signals 


\begin{tabular}{|c|c|}
\hline$s_{\mathrm{D}, \mathrm{SP}}$ & $\begin{array}{l}\text { Droplet length average calculated with the } \\
\text { syringe pump level }\end{array}$ \\
\hline SEM & Scanning electron microscopy \\
\hline SP & Syringe pump \\
\hline$T_{\mathrm{s} / \mathrm{s}}$ & $\begin{array}{l}\text { Transformation factor (diameter determined } \\
\text { with the syringe pump level to optical detector } \\
\text { signals) }\end{array}$ \\
\hline$\%(\mathrm{v} /$ & Volume/volume per cent \\
\hline$\%(\mathrm{w} /$ & Weight/volume per cent \\
\hline
\end{tabular}

\section{Introduction}

Microfluidic chips are used in droplet-based microfluidics to prepare serially arranged micro-reactors based on the immiscibility of at least two fluids (Chong et al. 2016). An increasing number of research groups use such micro-reactors for biological applications, e.g. for screening of microorganisms regarding their potential to produce pharmaceuticals (Zang et al. 2013) and to discover novel enzymes (Beneyton et al. 2016) or for investigating their resistance against heavy metals (Cao et al. 2013). Other groups reported about the cultivation of single cells in microfluidic droplets to detect their protein expression quantitatively (Huebner et al. 2007) or about screening and development studies on multicellular spheroids (McMillan et al. 2016) and even on embryos of multicellular organisms like the zebrafish (Funfak et al. 2007). Our group has recently reported about the dropletbased cultivation of embryoid bodies (EBs) formed from murine embryonic stem cells [mESCs (Lemke et al. 2015)]. These mESCs experiments were performed using the modularly constructed technological platform "pipe based bioreactors" (pbb) which has the potential to serve as long-term cultivation system for $3 \mathrm{D}$ cell cultures in the volume scale from $100 \mathrm{~nL}$ up to $10 \mu \mathrm{L}$ (Spitkovsky et al. 2016). Furthermore, droplet-based processes can be used to encapsulate pancreatic islets (Wiedemeier et al. 2011) and to analyse the quality of food (Schemberg et al. 2009, 2010).

An important prerequisite for the establishment of droplet-based applications is the availability of special devices for manipulating the droplets. One example is devices for the rapid and targeted sorting of droplets (Xi et al. 2017). Furthermore, new droplet generation concepts using different pressure conditions allow varying droplet sizes (Teo et al. 2017).

However, despite the huge potential of droplet-based microfluidics and the continuously growing number of publications in this field (Dressler et al. 2014), most studies only present proof-of-concepts (Casadevall i Solvas and deMello 2011; Volpatti and Yetisen 2014). One reason for this limited transferability to commercial/industrial applications is the restricted availability of disposable polymer chips that allow for a stable and reproducible droplet generation of challenging biologically relevant fluids with a high protein content (Shembekar et al. 2016).

Cyclic olefin copolymer (COC) (Mair et al. 2006) and polycarbonate (PC) (Sun et al. 2005) are well-established thermoplastic materials for microfluidic applications since they are optically transparent, sterilisable, easy to handle and economically priced. However, micro-channel surfaces of fluidic chips moulded from these polymers by standard injection moulding processes do not support a stable and reproducible droplet generation process. Especially in the case of generating droplets from biologically relevant fluids with a high protein concentration like cell culture media supplemented with serum, an adhesion of the droplets to the micro-channel surface and consequently droplet pinning resulting in cross-contamination are frequently observed. To avoid droplet pinning effects, micro-channel surfaces have to be modified to achieve superhydrophobic properties.

There are a lot of papers describing surface modifications to achieve superhydrophobic properties on polymers, e.g. (Shirtcliffe et al. 2011; Xue et al. 2010). Usually, the surface will be microstructured and hydrophobically coated. Well-established procedures are hot embossing (Wang et al. 2017), sol-gel coating (Wu et al. 2016) and deposition of soot (Esmeryan et al. 2017) or nanoparticles (Saarikoski et al. 2009). Furthermore, laser ablation procedures are used to structure the surfaces (Bachus et al. 2017; Rowthu et al. 2015). However, all these procedures are more expensive than producing microstructured chips by moulding or not suitable for droplet-based microfluidic applications. The final hydrophobisation is usually carried out by means of a wet chemical or plasma-supported coating (Jankowski et al. 2011).

In this study, we present a biomimetic approach to prepare anti-adhesive micro-channel surfaces on PC- and COC-chips with superhydrophobic lotus-leaf-like properties (Barthlott and Neinhuis 1997) for droplet-based microfluidic applications. The lotus effect is supposed to result from the combination of a hierarchical microtopography (i.e. papillae with a defined microstructure and nanostructure) and a hydrophobic epicuticular wax layer (Andreas et al. 2007; Darmanin and Guittard 2015; Quéré 2008). In order to replicate the lotus structure (Gornik 2004; Kim et al. 2013; Oh et al. 2011; Tuvshindorj et al. 2014), the surfaces of the moulds were equipped with a microtopography (Fig. 1) employing femtosecond pulsed laser ablation as described by Groenendijk (Groenendijk 2008).

After injection moulding, the chips were treated with a plasma coating procedure. The resulting surfaces were characterised topographically as well as physico-chemically. In order to analyse these microstructured and plasma-coated fluidic chips regarding their behaviour during and after droplet generation from aqueous media with a high protein concentration, fluidic experiments were performed. 
(a)
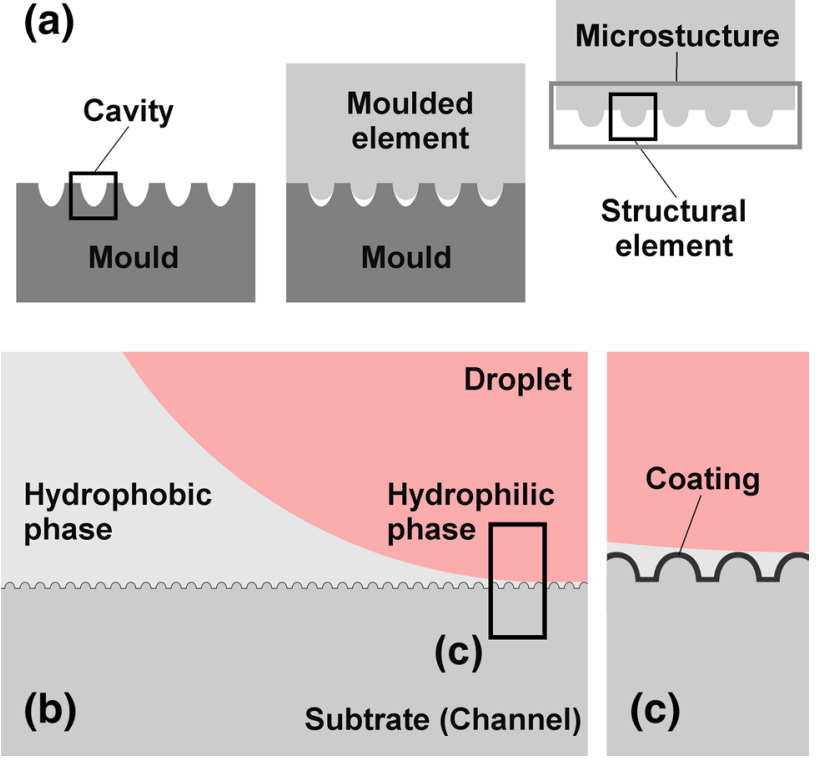

Fig. 1 a Schematic overview of the technological steps (from left to right, not drawn to scale). Left: laser-lithographically microstructured mould. Middle: moulding of the microstructure. Right: the moulded element with the microstructures and the structural elements. b Scheme of the three-phase system: microstructured, plasma-coated channel surface/hydrophobic phase (perfluorodecalin)/hydrophilic phase (droplet) and $\mathbf{c}$ scheme of the Cassie-Baxter wetting regime

The fluid micro systems (FMS chips) described in this paper were developed to handle cells and 3D cell structures like spheroids with diameters up to $500 \mu \mathrm{m}$. For this reason, the diameters of the droplet guiding channels were determined to be $1000 \mu \mathrm{m}$ resulting in droplet volumes not less than $523 \mathrm{~nL}$ to guarantee droplet contact to the circular channel walls. Droplet contact to the walls prevents merging of droplets. The smallest channel diameter for a reasonable manufacture by milling is proven to be $200 \mu \mathrm{m}$ resulting in droplet volumes of about $4 \mathrm{~nL}$. However, using moulding techniques, smaller channel diameters could be realised.

\section{Materials and methods}

\subsection{Preparation of microstructured moulds by laser ablation}

To investigate the chips surface properties on the one hand and their microfluidic properties on the other hand, two different chip types were moulded: (1) test elements (MST chips) without microfluidic channels but with a microstructured planar surface that were primarily employed for topographical and physico-chemical analyses (Fig. 1a) and (2) FMS chips with microstructured half-channels for droplet generation experiments (Fig. 4b, c). For these experiments, two of the half-channel possessing chips have to be assembled face-to-face resulting in circular cross sections of the microfluidic channels.

For the characterisation of unstructured surfaces, the reverse surfaces of the MST chips were used. The FMS chips were designed to perform $2 \mathrm{D}$ flow focussing experiments, i.e. each face-to-face assembled microfluidic chip possesses one main channel (diameter: $1000 \mu \mathrm{m}$ ) and two side channels (diameter: $300 \mu \mathrm{m}$ ), perpendicularly arranged to the main channel, see Fig. $4 \mathrm{~b}$. The size of a FMS chip is $24 \times 24 \times 4 \mathrm{~mm}^{3}$. The respective moulds were manufactured from hot working steel (1.2343). The semi-circular negative structures for moulding the half-channels were manufactured by electrical discharge machining.

Subsequently, surface microstructuring was performed by laser ablation using an ultrashort pulse laser (Hyper Rapid, Coherent GmbH, Germany) equipped with a Galvanometer scanner. The pulse width was 8 ps at a wavelength of $355 \mathrm{~nm}$. The laser power was $0.5 \mathrm{~W}$. For the mould of the MST chips, the microstructured area was $20 \times 20 \mathrm{~mm}^{2}$. For the FMS chip mould, only the negative structure for moulding the main channel and also the parts of the side channel that are in close proximity to the droplet generation zone were microstructured. A home-made tilting stage (ifw Jena, Germany) was employed to rotate the mould during the laser ablation process in order to obtain an angle of incidence of close to $90^{\circ}$ with respect to the curved surface of the semicircular negative structures of the mould. Both the tilting and the positioning of the samples demanded a high degree of precision ( $\mu \mathrm{m}$ scale).

Inspired by the microstructure of the anti-adhesive lotus leaves, micro-cavities with a depth of $10-15 \mu \mathrm{m}$ and a spacing of $10-15 \mu \mathrm{m}$ were created by laser ablation (Groenendijk and Meijer 2006). The diameter of the laser focus was $10 \mu \mathrm{m}$. The depths of the micro-cavities were adjusted by varying the density of the laser pulses per area (repetitions or pulse count). To achieve a high surface density, the microcavities were arranged in a hexagonal pattern (Andreas et al. 2007; Feng et al. 2008).

\subsection{Injection moulding and coating}

COC (COC615) and two PC materials (PC2400 and PC2805) with different viscosities were used as chip moulding polymers. The viscosity increased from PC2400 < PC2805 < COC615. The chips were manufactured by injection moulding (Allrounder 320 s, Arburg, Germany) with the following process parameters (Table 1).

After injection moulding, the chips were cleaned by treatment with isopropanol for $15 \mathrm{~min}$ in an ultrasonic bath (Sonorex super RK $100 \mathrm{H}$, Bandelin electronic GmbH \& Co. KG, Germany) and successive rinsing with ethanol $[80 \%(\mathrm{v} / \mathrm{v})]$ and deionised water. 
Table 1 Injection-moulding parameter

\begin{tabular}{lll}
\hline Parameter & Value & Unit \\
\hline Melting temperature & 320 & ${ }^{\circ} \mathrm{C}$ \\
Injection pressure & 45.3 & $\mathrm{MPa}$ \\
Injection velocity & 20 & $\mathrm{ml} / \mathrm{s}$ \\
Injection time & 0.9 & $\mathrm{~s}$ \\
Dosing time & 2.35 & $\mathrm{~s}$ \\
Cooling time & 70 & $\mathrm{~s}$ \\
Dwell pressure & 160 & $\mathrm{MPa}$ \\
Moulding temperature & 150 & ${ }^{\circ} \mathrm{C}$ \\
\hline
\end{tabular}

Subsequently, the surfaces of the chips were coated with a hydrophobic layer employing a low-pressure plasma system (Pico 110265, Type F, Diener electronic GmbH + Co. KG, Germany). After evacuating the reaction chamber down to a pressure of $9 \mathrm{~Pa}$, the octafluorocyclobutane precursor $\left(\mathrm{C}_{4} \mathrm{~F}_{8}\right.$, Air Liquide, Germany) was injected with $15 \mathrm{sccm}$, and the plasma was initiated at $13.56 \mathrm{MHz}$. Plasma coating was performed for $30 \mathrm{~min}$. The coated chips were stored until use in a closed chamber.

\subsection{Topographical surface characterisation}

\subsubsection{Scanning electron microscopy (SEM)}

After sputter coating with an approx. $9 \mathrm{~nm}$ gold layer (K550X Sputter Coater, Quorum Technologies Ltd, UK), the microstructured surface topography of the MST chips was characterised by means of stereo scanning electron microscopy (Evo LS10, Carl Zeiss Microscopy GmbH, Germany). SEM images from three tilt angels $\left(0^{\circ}-7^{\circ}-15^{\circ}\right)$ were recorded with a 250 fold magnification, $9 \mathrm{~mm}$ working distance and 1024 pixel $\times 768$ pixel resolution. From these SEM images, a three-dimensional, digital surface model (3D-DSM) was composed employing the $\mathrm{MeX}^{\circledR}$ software (Alicona Imaging $\mathrm{GmbH}$, Austria). The analysis tool of the same software was used to determine the depths of the micro-cavities.

\subsubsection{Atomic force microscopy (AFM)}

AFM investigations on MST chips were performed using the atomic force microscope Nanowizard ${ }^{\circledR}$ equipped with a $100 \mu \mathrm{m}$ z-scan module CellHesion ${ }^{\circledR}$ (both jpk instruments AG, Germany). All scans were performed in contact mode at ambient conditions with cantilevers ARROW-NC (NanoWorld AG, Switzerland) having a nominal spring constant of $42 \mathrm{~N} / \mathrm{m}$ and a tip radius less than $20 \mathrm{~nm}$. Due to the high aspect ratio of the micro-cavities, the scans were performed with a low scan rate of $0.1 \mathrm{~Hz}$. In addition to the analyses of the microstructured chips, the hierarchical topography of a lotus leave (Nelumbo nucifera) was investigated, as well. The lotus leaf was stabilised with glycerol to avoid drying artefacts during the scans. Post-processing and 3D images of data were realised using the background correction feature and a Gaussian smoothing filter of the software SPIPTM (Image Metrology A/S, Denmark).

\subsection{Physico-chemical characterisation}

Surface free energy determinations (including polar and dispersive components) were performed on the microstructured MST chips before and after the plasma coating procedure according the OWRK approach (Owens, Wendt, Rabel und Kaelble). For this approach, the contact angles of deionised water, formamide, ethylene glycol (predominantly polar) and diiodomethane (dispersive) were recorded using the OCA System (sessile drop, 3 droplets of $3 \mu \mathrm{L}$, DataPhysics Instruments GmbH, Germany).

\subsection{Fluidic experiments}

Fluidic experiments were performed with a highly versatile microfluidic platform comprising the following functional modules:

- Droplet generation FMS,

- Detection module (equipped with two optical sensors),

- Tube storage disc,

- Stirring unit (only for experiments with blood),

- Syringe pump system (neMESYS, cetoni GmbH, Germany).

The droplet generation FMS is composed of two plasmacoated chip halves assembled reversibly face-to-face and pressed together with screws guaranteeing leak tightness (Gastrock et al. 2010). Pins and drillings serve as positioning elements to guarantee for a reproducible assembly and disassembly, e.g. for refreshing of the chip coating. After its assembling, the FMS is mounted into a frame that serves for the fluidic connections between the tubes and the FMS (Fig. 4a). All functional modules were connected with tubes made from polytetrafluoroethylene (PTFE) and fluorinated ethylene propylene (FEP), respectively.

For both, the transport of droplets as well as the mutual separation of the droplets, perfluorodecalin (PFD) served as continuous phase. Additionally, reference experiments with Novec 7500 and Pico-Surf ${ }^{\mathrm{TM}} 2$ as continuous phase were performed. Two aqueous fluids with a high biotechnological and biomedical relevancy were used for the experiments performed in this study: (1) cell culture medium Dulbecco's Modified Eagle's Medium (DMEM), product number D5523 (Sigma-Aldrich Chemie GmbH, Germany) supplemented with $4.5 \mathrm{~g} / \mathrm{L}$ D-glucose, $2 \mathrm{mmol} / \mathrm{l}$ L-glutamine, 
$100 \mathrm{U} / \mathrm{mL}$ penicillin, $100 \mu \mathrm{g} / \mathrm{mL}$ streptomycin, $10 \% \mathrm{v} / \mathrm{v}$ foetal calf serum and $0.01 \% \mathrm{w} / \mathrm{v}$ phenol red and (2) anticoagulated human blood (supplemented with trisodium citrate $0.106 \mathrm{~mol} / \mathrm{L}$ in S-Monovette ${ }^{\circledR}$, order number 04.1955.100, Sarstedt AG \& Co., Germany). For droplet-based microfluidic experiments, those fluids are often challenging due to their high concentration of surface active compounds like proteins. In order to characterise the influence of the FMS surface microstructure and coating on the droplet generation process, most experiments were performed without surfactants. However, as reference to usual droplet-based applications, also experiments with surfactants were performed.

Prior to the experiments, the droplet generation FMS and the tubes were intensively rinsed and filled with PFD. For each parameter, five experiments were performed with a PFD flow rate $\left(Q_{\mathrm{c}}\right)$ of $500 \mu \mathrm{L} / \mathrm{min}$, an aqueous fluid flow rate $\left(Q_{\mathrm{d}}\right)$ of $100 \mu \mathrm{L} / \mathrm{min}$ and a droplet generation time of $6 \mathrm{~min}$.

To evaluate the droplet generation reproducibility, the volume of each droplet and its standard deviation were assessed. Each droplet was considered as a sphere (sphere diameter $\leq$ main channel diameter of the droplet generation FMS) or as cylinder with hemispherical ends. In the latter case, the cylinder diameter corresponds to the main channel diameter of the droplet generation FMS.

For the cylinder volume calculation, the droplet length was measured photometric at $525 \mathrm{~nm}$ by recording the absorption shift between PFD and the droplet. These measurements were realised using a detection chip with two optical sensors.

The droplet volume calculation bases on the precondition of an idealised droplet shape. However, depending on the droplet velocity, deviations from these idealised shapes were observed. Compared to a non-moving (nondeformed) droplet, the shape deviation became more significant with increasing velocity. Taking these shape deviations into account, a correction factor was introduced. This factor $T_{\mathrm{s} / \mathrm{s}}$ represents the ratio of the droplet length average $s_{\mathrm{D}, \mathrm{SP}}$, calculated from the sample volume delivered by the syringes pump (SP) divided by the number of generated droplets and the droplet length average $s_{\mathrm{D}, \mathrm{OS}}$, determined by the optical sensors (OS).

$T_{\mathrm{s} / \mathrm{s}}=\frac{s_{\mathrm{D}, \mathrm{SP}}}{s_{\mathrm{D}, \mathrm{OS}}}$

Subsequently, the volume of each droplet was calculated employing this correction factor.

To evaluate the droplet generation reproducibility, the following parameters were determined for each experiment: (1) the mean droplet volume, (2) the standard deviation and (3) the coefficient of variation (CV).

For the final analysis of the droplet generation stability, two further experiments were performed. Within the scope of these experiments, interactions of the sample medium with the FMS channel surface should be investigated. For this, droplets were continuously generated for $6 \mathrm{~h}$ using the FMS chips to be examined. The evaluation was based on the pure optical observation of the droplet generation process. The experiment was stopped when the droplets extensively adhered to the channel surface, and the stability of droplet generation process was significantly disturbed.

\section{Results and discussion}

\subsection{Influence of the microstructure on the physico-chemical properties}

With the applied injection-moulding process, papilla-like structures could successfully be prepared on different polymers surfaces (Fig. 2a-c).

The use of different polymers resulted in different morphologies of the papilla-like structures. The flattest papillae were observed on the surface of MST chips made from COC (Fig. 2c), whereas the PC2805 surfaces were characterised by rather sharp papillae (Fig. 2b).

The different polymers also caused different heights of the papillae; i.e. heights of $\sim 5 \mu \mathrm{m}$ for PC2805 (Fig. 2b) and COC6017 (Fig. 2c) and heights of $\sim 6 \mu \mathrm{m}$ for PC2400 (Fig. 2a). These differences are caused by the different viscosities of the molten polymers. A lower viscosity of the melt improves its intrusion into the mould cavities and consequently increases the height of the papillae. The polymer PC2400 with the lowest viscosity (PC2400 < PC2805 < COC6017) resulted in the maximum papillae height.

Compared to unstructured and uncoated polymer MST chip surfaces, the contact angles of uncoated microstructured MST chip surfaces increased from $\sim 90^{\circ}$ to $\sim 120^{\circ}$ (Fig. 2d). The highest water contact angle on uncoated but microstructured surfaces was measured with $\sim 130^{\circ}$ on the COC6017 MST surface. This also connotes that microstructured COC6017 FMS should be well suited for droplet-based applications even without plasma coating.

The surface free energy of the unstructured and uncoated MST surfaces was $\sim 40 \mathrm{mN} / \mathrm{m}$ (Fig. 2e). Caused by the microstructure, the surface free energy was increased by $\sim 6 \mathrm{mN} / \mathrm{m}$ for the MST chips with smaller papillae height (PC2805 and COC6017) and by $\sim 20 \mathrm{mN} / \mathrm{m}$ for the MST chips with maximum papillae height (PC2400, Fig. 2e).

In the case of unstructured surfaces of MST chips made from the polymers PC2400 and PC2805, 95\% of the surface free energy was contributed by the dispersive component. For COC6017, the dispersive component was $~ 89 \%$. For the microstructured surface of MST chips, the dispersive 

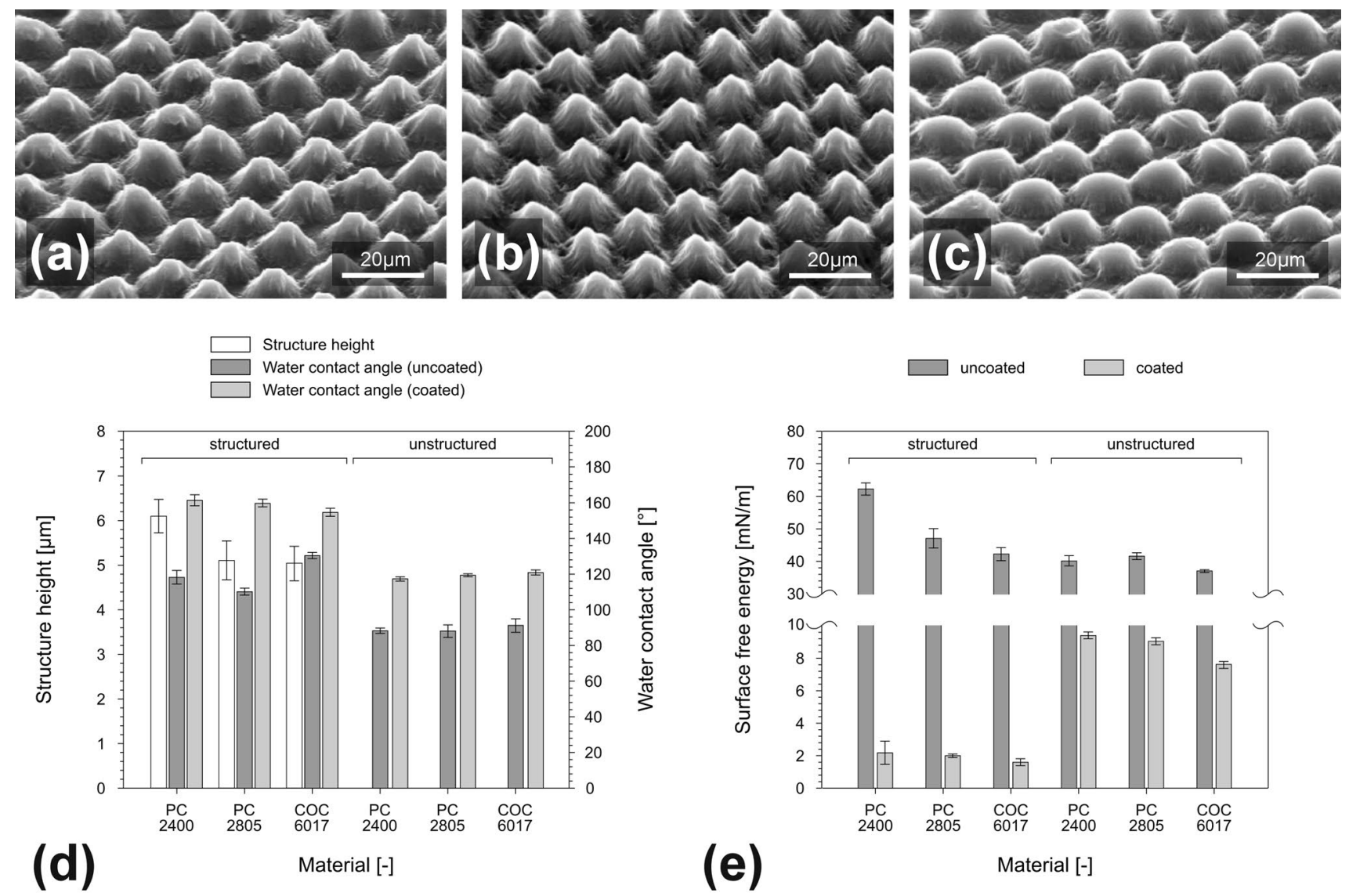

Fig. 2 Upper panels: SEM images $\left(45^{\circ}\right.$ angle) of the microstructured moulded polymers PC2400 (a), PC2805 (b) and COC6017 (c). Lower panels: comparison of uncoated and coated as well as unstructured

and structured moulded surfaces. d Results of the quantitative analyses of the heights of the created microstructural elements and resulting water contact angle and $\mathbf{e}$ free surface energy of the polymers

component was increased by 3\% for PC2400 and PC2805 and by $\sim 10 \%$ for COC6017.

In summary, the uncoated microstructure of the MST chip surfaces increases both the water wettability and the surface free energy. This phenomenon was described by Wenzel (Wenzel 1949): for a homogeneous wetting scenario where microstructures intensify the surface properties of the bulk material (hydrophobic solid materials become more hydrophobic and vice versa).

\subsection{Influence of the plasma coating on the physico-chemical properties}

Plasma coating resulted in a significant increase of the water contact angle for both the unstructured and the microstructured MST surfaces (Fig. 2d, coated surfaces). The surface of an unstructured MST chips moulded from PC2805 revealed a water contact angle of $88.1^{\circ} \pm 3.4^{\circ}$ and a surface free energy of $41.74 \pm 1.0 \mathrm{mN} / \mathrm{m}$ (dispersive: $41.56 \pm 1.1 \mathrm{mN} / \mathrm{m}$, polar: $1.9 \pm 0.3 \mathrm{mN} / \mathrm{m}$ ). After plasma coating with $\mathrm{C}_{4} \mathrm{~F}_{8}$, the wettability was significantly affected.

The water contact angle increased to $119.4^{\circ} \pm 0.7^{\circ}$, and the surface free energy was reduced to $8.98 \pm 0.2 \mathrm{mN} / \mathrm{m}$ (dispersive: $8.44 \pm 0.3 \mathrm{mN} / \mathrm{m}$, polar: $0.54 \pm 0.2 \mathrm{mN} / \mathrm{m}$ ).

Interestingly, the wettability was not only reduced for aqueous test fluids but also for non-fluorinated oils like tetradecane. However, the coated surfaces were completely wettable with fluorinated oils like PFD (contact angle $<20^{\circ}$ ).

From AFM measurements, the thickness of the $\mathrm{C}_{4} \mathrm{~F}_{8}$-coating was estimated to be $\sim 140 \mathrm{~nm}$. For this, a glass slide was partially coated using the above-described coating procedure.

For the microstructured surfaces, the water contact angle increased from $\sim 110^{\circ}$ to $\sim 130^{\circ}$ up to $\sim 160^{\circ}$ after plasma coating. Furthermore, the standard deviation of the water contact angles was reduced for all coated MST chips. After plasma coating the wettability of the three polymers were comparable even though there were significant differences in the wettability prior to the coating process (Fig. 2d). The polymer with the highest contact angle before coating $\left(130^{\circ}\right.$ for microstructured COC6017) displayed the lowest contact angle after plasma 
coating $\left(154^{\circ}\right)$. Since the structure heights of PC2805 and COC6017 (Fig. 2d) are comparable, we assume that the rounded morphology of the COC6017 microstructure elements (Fig. 2c) causes an increase of the contact area to the polar phase resulting in a slightly increased wettability.

In addition to the reduction of the water wettability, a significant reduction of the surface free energy was observed after coating both microstructured and not microstructured MST surfaces (Fig. 2e). For the unstructured surfaces, the surface free energy was reduced by more than $30 \mathrm{mN} / \mathrm{m}$ to values around $9 \mathrm{mN} / \mathrm{m}$. An extremely low surface free energy of $\sim 2 \mathrm{mN} / \mathrm{m}$ was observed for the microstructured surfaces after plasma coating. This reduction is predominantly due to a decrease of the dispersive part of the surface free energy. While the coating procedure does not significantly affect the polar component for unstructured surfaces $(\sim 1 \mathrm{mN} / \mathrm{m})$, it is increased to $\sim 4 \mathrm{mN} / \mathrm{m}$ for uncoated microstructured samples. When these microstructured surfaces are plasma coated, the polar part is reduced to values lower than $0.2 \mathrm{mN} / \mathrm{m}$.

In general, polar components account for less than $10 \%$ of the surface free energy irrespective of the coating. The high water contact angles additionally indicate a low contribution of the polar part of the surface free energy, especially since the coated microstructured polymer surfaces display an extremely low polar contribution.

\subsection{Comparison of the coated microstructured polymer surfaces with the lotus leaf surface}

In order to compare the technically prepared biomimetic surfaces with the leaves of the lotus flower, AFM analyses were performed on microstructured and plasma-coated PC2400 MST chips and Nelumbo nucifera leaves. Comparative AFM analyses reveal that both surfaces are characterised by a hierarchical micro- and nanotopography (Fig. 3). The morphology of the individual microstructure elements of the injection-moulded polymer surface reveals a striking similarity to the papillae of the lotus leaf (Fig. 3b, e). They also display comparable heights, diameters and spacing.

The arrangement of the microstructure elements is characterised by a lower degree of variability (in terms of height and spacing of individual microstructure elements, Fig. 3a, d). Furthermore, both surfaces display a similar nanotopography on top of the microstructure elements (Fig. 3c, f).

Both surfaces reveal an extremely low water wettability $\left(\sim 160^{\circ}\right.$ for the lotus leaf (Barthlott and Neinhuis 1997) as well as for the microstructured, plasma-coated polymer surface). These superhydrophobic properties indicate a heterogeneous wetting according to the Cassie-Baxter theory (Cassie and Baxter 1944; Hüger et al. 2009; Wagner et al. 2003).
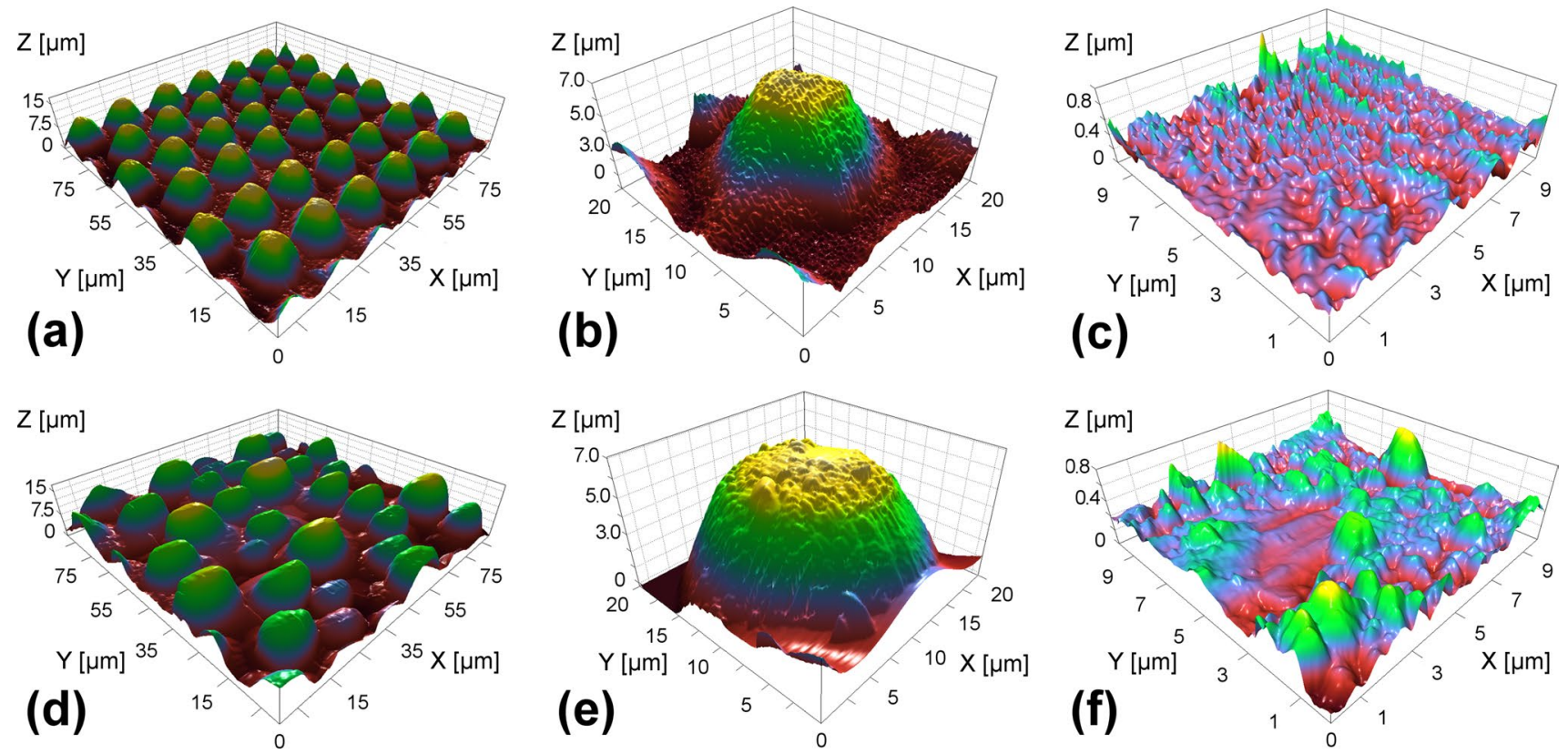

Fig. 3 AFM analyses on the topography of microstructured, plasmacoated MST surfaces (a-c, PC2400) and a Nelumbo nucifera leaf (d-f). The images reveal the striking similarities between the two hierarchical structures on different scales: arrangement of the micro-

structure elements (a and d), morphology of the individual microstructure elements (b and e) and the nanotopography on top of the microstructure elements (c and $\mathbf{f}$ ) 

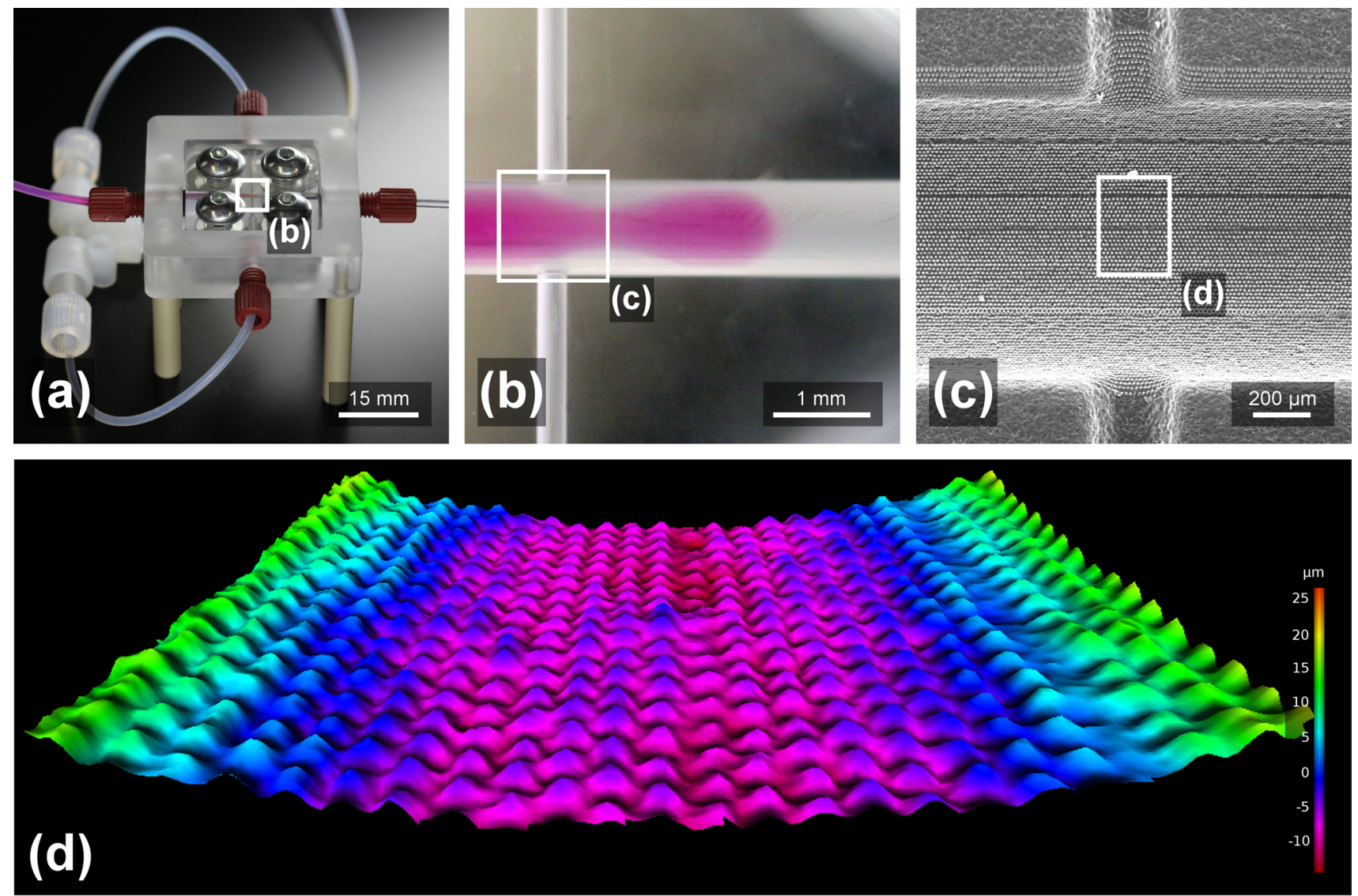

117. Water contact angle (structured)

VIII Surface free energy (structured)

$\square$ Water contact angle (unstructured)

$\square$ Surface free energy (unstructured)
$\square$

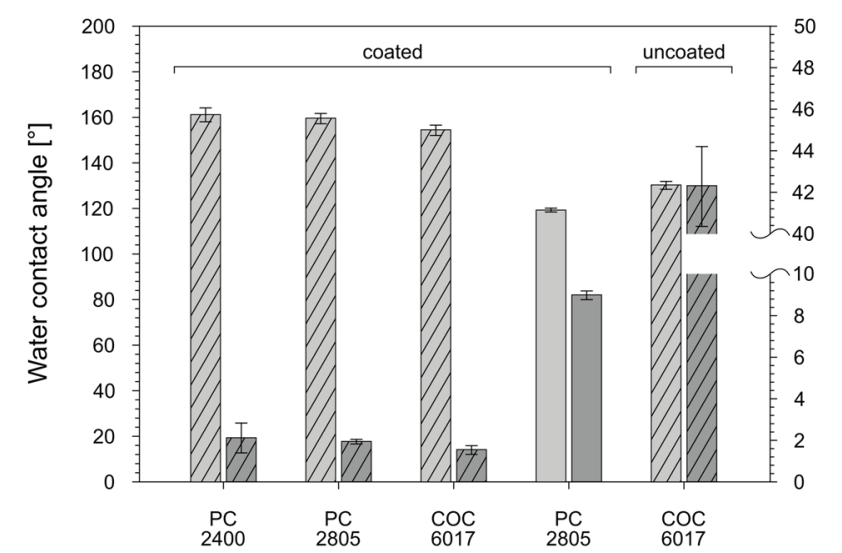

(e)

Material [-]

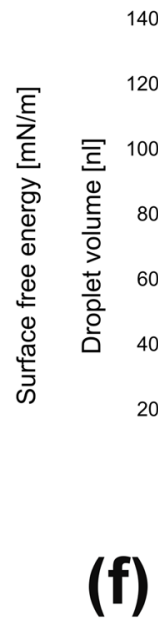

$\square / /$ Droplet volume (structured)

$\square$ Droplet volume (unstructured)

Fig. 4 FMS chip with microstructured, plasma-coated channels prepared by injection moulding of PC2805 a photograph of the FMS mounted in a frame for fluidic contacting, b photograph and $\mathbf{c}$ SEM image of the intersection between main channel (aqueous phase) and

\subsection{Microfluidic experiments}

The FMS microstructure was created on the surface of the

side channel (separation medium), d 3D digital surface model of the microstructured channel surface, $\mathbf{e}$ physico-chemical properties and $\mathbf{f}$ results of the microfluidic experiments for FMS prepared from different polymers (see also Table 2)

main channel which is in direct contact with the test fluids. SEM and stereoSEM images of the main channel surface reveal that the microstructure that was created on the mould 
could be successfully transferred onto the FMS chip elements (Fig. 4c, d). For the microfluidic experiments with DMEM and blood, the microstructured and plasma-coated FMS chips made from the above-mentioned polymers were investigated (Table 2, No. 1-5). Additionally, reference FMS chips (Table 2, No. 6-7) were investigated to estimate the effect of a missing microstructure (unstructured PC2805) as well as of a missing $\mathrm{C}_{4} \mathrm{~F}_{8}$ coat (uncoated COC6017).

Irrespective of the type of polymer, no significant adhesion of the cell culture medium to the channel surface was detected during the $30 \mathrm{~min}$ of droplet generation employing coated microstructured FMS (Table 2, No. 2-5). However, severely reduced performance times of $\sim 10 \mathrm{~min}$ for the unstructured but plasma-coated PC2805 reference FMS (Table 2, No. 6) and $~ 15$ min for the microstructured but uncoated COC6017 reference FMS (Table 2, No. 7) were observed. This indicates that a combination of both the microstructure and the plasma coating is necessary to guarantee for a stable droplet generation process.

In addition to these effects, the surface modification also affects both the droplet volume and the reproducibility of the droplet generation process (Fig. 4f). When the droplet generation was performed with microstructured and coated FMS, a consistent droplet volume of $\sim 800 \mathrm{~nL}$ was obtained. For all microstructured and coated FMS investigated with DMEM (Table 2, No. 3-5), there is a slight tendency of an increasing droplet volume for a decreasing water contact angle (and a decreasing surface free energy), compare Fig. 4 e, f.

For the unstructured channel surface of the PC2805 FMS (Table 2, No. 6), the droplet volumes increased by $\sim 200 \mathrm{~nL}$ (+25\%), see Fig. 4e, f. A similar effect was observed for the microstructured but not plasma-coated COC6017 FMS (Table 2, No. 7). The droplet volumes increased to $\sim 1100 \mathrm{~nL}$ ( + 35\%) when uncoated COC6017 FMS were employed (Fig. 4e, f). Each of these droplet volume increases correlates with a higher wettability of the surfaces $\left(\sim 30^{\circ}\right.$ lower

Table 2 FMS chip configurations and the investigated fluids

\begin{tabular}{llllll}
\hline No. & Polymer & Microstructure & Coating & Test fluid & $\begin{array}{c}\text { Continu- } \\
\text { ous phase }\end{array}$ \\
\hline 1 & PC2400 & Yes & Yes & Blood & PFD \\
2 & PC2400 & Yes & Yes & DMEM & $\begin{array}{c}\text { Novec } \\
7500\end{array}$ \\
& & & & & and \\
& & & & & Pico- \\
& & & & & surf 2 \\
3 & PC2400 & Yes & Yes & DMEM & PFD \\
4 & PC2805 & Yes & Yes & DMEM & PFD \\
5 & COC6017 & Yes & Yes & DMEM & PFD \\
6 & PC2805 & No & Yes & DMEM & PFD \\
7 & COC6017 & Yes & No & DMEM & PFD \\
\hline
\end{tabular}

water contact angles and higher surface free energy, Fig. 4e, f). The higher wettability of the FMS channel surface causes more pronounced interactions of DMEM with the channel surface, resulting in a delayed droplet break-up and thus in an increase of the droplet volume.

For all microstructured and plasma-coated FMS, the CV values were smaller than $2.5 \%$, indicating that the droplet generation process was highly reproducible. The lowest CV value of $1.45 \%$, and thus, the highest reproducibility was observed for the microstructured and plasma-coated COC6017 FMS.

For droplet generation from anticoagulated human whole blood, a coated microstructured PC2400 FMS was used (Table 2, No. 1). Droplets could stably be generated during the whole process, lasting 30 min maximally. In contrast to the experiments with DMEM, droplets from blood could not be generated with microstructured but uncoated PC FMS. The blood droplet volumes and their $\mathrm{CV}$ values were higher than the respective data for droplets generated from DMEM. This may be due to the higher protein concentration of whole blood that supports interactions with the uncoated channel surface (Pham et al. 2016).

Compared to the experiments without surfactants (Table 2, No. 3), the results of the reference experiments with the PC 2400 FMS using surfactants (Table 2, No. 2) show a droplet volume increase of approx. $22 \%$, see also Fig. 4f. This volume increase is caused by the decreased surface tension. The $\mathrm{CV}$ value increased merely by about $0.2 \%$.

Finally, the experiments with DMEM and whole blood were continued with the four structured and coated FMS (Table 2, No. 1-5) to study the long-term stability of the droplet generation. For this purpose, droplets were continuously generated on two successive days each for $6 \mathrm{~h}$ using the corresponding FMS as well as the test fluids DMEM and whole blood. Using three different, microstructured and coated FMS (Table 2, No. 2-5), droplets could be generated without disturbance over the complete test period of $12 \mathrm{~h}$ with DMEM. In the case of droplet generation from whole blood using the microstructured and coated PC2400 MST (Table 2, No. 1), punctiform adhesions of the blood on the channel surface were observed after approx. $10 \mathrm{~h}$. However, these adhesions could be eliminated by increasing the flow rates and did not appear again. These experiments proved that injection-moulded, microstructured and plasma-coated FMS support a stable and long-term droplet generation process.

In summary, both the introduced microstructure and the hydrophobic plasma coating lower the surface free energy of the FMS channel surfaces. Consequently, the tendency of biologically relevant fluids to adhere to the channel surface is lowered. According to Cassie's law (Cassie and Baxter 1945; Cassie 1948), the contact area between the droplets and the channel surface could be significantly reduced. Particularly 
for hydrophobic surfaces (Absolom et al. 1987), this reduced contact area should also reduce the capability for protein adhesion (Fig. 1b, c). A combination of the microstructure and the hydrophobic plasma coating significantly reduces the adhesion of biologically relevant fluids to the channel surface. The resulting disposable FMS chips support a long-term stable droplet generation process with a highly reproducible droplet volume.

\section{Conclusions}

The results presented in this study demonstrate that microfluidic chips with biomimetic anti-adhesive channel surfaces can be prepared by combining injection moulding and plasma coating. Injection moulding was performed in moulds that were microstructured via laser ablation. The resulting superhydrophobic surfaces reveal a hierarchical microtopography and physico-chemical properties similar to the lotus leaf. Dropletbased microfluidic experiments reveal that only the combination of the microtopography and the anti-adhesive $\mathrm{C}_{4} \mathrm{~F}_{8}$-plasma coating supports a stable long-term droplet generation process. Droplets could even be prepared from challenging fluids like whole blood with a high protein concentration in a stable and highly reproducible fashion. In combination with the modular platform (iba 2005; Lemke et al. 2015), the FMS chips possess a high potential for commercial, droplet-based applications in life sciences since they support a stable and reproducible droplet generation process and since they can be prepared as sterilisable disposables.

The advantage of the approach for increasing the hydrophobicity of polymer surfaces proposed in this paper is the direct microstructure transfer from the mould to the chip, followed by a plain plasma coating.

Compared to the manufacture of ordinary moulds, only one additional manufacturing step is necessary. After milling and electroerosion of the mould, the microstructures were realised by laser ablation. During the moulding process, these microstructures were transferred onto the FMS chips. After coating the FMS chips with plasma, two chip halves were mounted face-to-face to achieve round channels. After mounting the FMS chips, they can be used for the experiments. Laser ablation is the only additional manufacture step.

Acknowledgements Financial support from Thüringer Aufbaubank (TAB, EFRE Grant Number 2013 VF 0003) is gratefully acknowledged. The authors thank Anja Körner and Johannes Drosten for technical support.

Open Access This article is distributed under the terms of the Creative Commons Attribution 4.0 International License (http://creativecommons.org/licenses/by/4.0/), which permits unrestricted use, distribution, and reproduction in any medium, provided you give appropriate credit to the original author(s) and the source, provide a link to the Creative Commons license, and indicate if changes were made.

\section{References}

Absolom DR, Zingg W, Neumann AW (1987) Protein adsorption to polymer particles: role of surface properties. J Biomed Mater Res 21:161-171. doi:10.1002/jbm.820210202

Andreas S, Zdenek C, Boris FS, Manuel S, Wilhelm B (2007) The dream of staying clean: lotus and biomimetic surfaces. Bioinspir Biomim 2:S126

Bachus KJ, Mats L, Choi HW, Gibson GTT, Oleschuk RD (2017) Fabrication of patterned superhydrophobic/hydrophilic substrates by laser micromachining for small volume deposition and dropletbased fluorescence. ACS Appl Mater Interfaces 9:7629-7636. doi:10.1021/acsami.6b16363

Barthlott W, Neinhuis C (1997) Purity of the sacred lotus, or escape from contamination in biological surfaces. Planta 202:1-8

Beneyton $T$ et al (2016) High-throughput screening of filamentous fungi using nanoliter-range droplet-based microfluidics. Sci Rep 6:27223. doi: $10.1038 /$ srep27223

Cao J, Kürsten D, Krause K, Kothe E, Martin K, Roth M, Köhler JM (2013) Application of micro-segmented flow for two-dimensional characterization of the combinatorial effect of zinc and copper ions on metal-tolerant Streptomyces strains. Appl Microbiol Biotechnol 97:8923-8930. doi:10.1007/s00253-013-5147-8

Casadevall i Solvas X, deMello A (2011) Droplet microfluidics: recent developments and future applications. Chem Commun 47:19361942. doi:10.1039/c0cc02474k

Cassie ABD (1948) Contact angles. Discuss Faraday Soc 3:11-16. doi:10.1039/DF9480300011

Cassie ABD, Baxter S (1944) Wettability of porous surfaces. Trans Faraday Soc 40:546-551. doi:10.1039/TF9444000546

Cassie A, Baxter S (1945) Large contact angles of plant and animal surfaces. Nature 155:21-22

Chong ZZ, Tan SH, Ganan-Calvo AM, Tor SB, Loh NH, Nguyen N-T (2016) Active droplet generation in microfluidics. Lab Chip 16:35-58. doi:10.1039/C5LC01012H

Darmanin T, Guittard F (2015) Superhydrophobic and superoleophobic properties in nature. Mater Today 18:273-285. doi:10.1016/j. mattod.2015.01.001

Dressler OJ, Maceiczyk RM, Chang S-I, deMello AJ (2014) Dropletbased microfluidics enabling impact on drug discovery. J Biomol Screen 19:483-496. doi:10.1177/1087057113510401

Esmeryan KD, Castano CE, Mohammadi R (2017) Interactions of superhydrophobic carbon soot coatings with short alkyl chain alcohols and fluorocarbon solutions. Colloids Surf A 529:715724. doi:10.1016/j.colsurfa.2017.06.067

Feng L, Zhang Y, Xi J, Zhu Y, Wang N, Xia F, Jiang L (2008) Petal effect: a superhydrophobic state with high adhesive force. Langmuir 24:4114-4119. doi:10.1021/la703821h

Funfak A, Brosing A, Brand M, Kohler JM (2007) Micro fluid segment technique for screening and development studies on Danio rerio embryos. Lab Chip 7:1132-1138. doi:10.1039/b701116d

Gastrock G, Grodrian A, Lemke K, Wiedemeier S, Römer R (2010) Mountable and dismountable microfluid system and method for flooding the system. Google Patents

Gornik C (2004) Injection moulding of parts with microstructured surfaces for medical applications. Macromol Symp 217:365-374. doi:10.1002/masy.200451332

Groenendijk M (2008) Fabrication of super hydrophobic surfaces by fs laser pulses. Laser Technik J 5:44-47. doi:10.1002/latj.200890025

Groenendijk MNW, Meijer J (2006) Surface microstructures obtained by femtosecond laser pulses. CIRP Ann Manuf Technol 55:183186. doi:10.1016/S0007-8506(07)60394-1

Huebner A, Srisa-Art M, Holt D, Abell C, Hollfelder F, deMello AJ, Edel JB (2007) Quantitative detection of protein expression 
in single cells using droplet microfluidics. Chem Commun. doi:10.1039/b618570c

Hüger E, Rothe H, Frant M, Grohmann S, Hildebrand G, Liefeith K (2009) Atomic force microscopy and thermodynamics on taro, a self-cleaning plant leaf. Appl Phys Lett 95:033702. doi:10.1063/1.3184582

iba (2005) Mark Trade Mark pipe-based bioreactors (pbb):Reg. no. 305304226

Jankowski P, Ogonczyk D, Kosinski A, Lisowski W, Garstecki P (2011) Hydrophobic modification of polycarbonate for reproducible and stable formation of biocompatible microparticles. Lab Chip 11:748-752. doi:10.1039/C0LC00360C

Kim BI et al (2013) A continuous tilting of micromolds for fabricating polymeric microstructures in microinjection. Lab Chip 13:43214325. doi:10.1039/c3lc50494h

Lemke K, Förster T, Römer R, Quade M, Wiedemeier S, Grodrian A, Gastrock G (2015) A modular segmented-flow platform for 3D cell cultivation. J Biotechnol 205:59-69. doi:10.1016/j. jbiotec.2014.11.040

Mair DA, Geiger E, Pisano AP, Frechet JMJ, Svec F (2006) Injection molded microfluidic chips featuring integrated interconnects. Lab Chip 6:1346-1354. doi:10.1039/b605911b

McMillan KS, Boyd M, Zagnoni M (2016) Transitioning from multiphase to single-phase microfluidics for long-term culture and treatment of multicellular spheroids. Lab Chip 16:3548-3557. doi:10.1039/C6LC00884D

Oh HJ, Park JH, Lee SJ, Kim BI, Song YS, Youn JR (2011) Sustainable fabrication of micro-structured lab-on-a-chip. Lab Chip 11:39994005. doi:10.1039/C1LC20441F

Pham TT et al (2016) Hemodynamic aspects of reduced platelet adhesion on bioinspired microstructured surfaces. Colloids Surf B 145:502-509. doi:10.1016/j.colsurfb.2016.05.022

Quéré D (2008) Wetting and roughness. Annu Rev Mater Res 38:7199. doi:10.1146/annurev.matsci.38.060407.132434

Rowthu S, Böhlen K, Bowen P, Hoffmann P (2015) Surface 3D micro free forms: multifunctional microstructured mesoporous $\alpha$-alumina by in situ slip casting using exclaimer laser ablated polycarbonate molds. ACS Appl Mater Interfaces 7:24458-24469. doi:10.1021/acsami.5b04748

Saarikoski I, Suvanto M, Pakkanen TA (2009) Modification of polycarbonate surface properties by nano-, micro-, and hierarchical micro-nanostructuring. Appl Surf Sci 255:9000-9005. doi:10.1016/j.apsusc.2009.06.073

Schemberg J, Grodrian A, Römer R, Gastrock G, Lemke K (2009) Online optical detection of food contaminants in microdroplets. Eng Life Sci 9:391-397. doi:10.1002/elsc.200800127

Schemberg J, Grodrian A, Römer R, Cahill BP, Gastrock G, Lemke K (2010) Application of segmented flow for quality control of food using microfluidic tools. Phys Status Solidi (A) 207:904-912. doi:10.1002/pssa.200983315

Shembekar N, Chaipan C, Utharala R, Merten CA (2016) Dropletbased microfluidics in drug discovery, transcriptomics and high-throughput molecular genetics. Lab Chip 16:1314-1331. doi:10.1039/c6lc00249h

Shirtcliffe NJ, McHale GI, Newton M (2011) The superhydrophobicity of polymer surfaces: recent developments. J Polym Sci B Polym Phys 49:1203-1217. doi:10.1002/polb.22286

Spitkovsky D et al (2016) Generation of cardiomyocytes in pipe-based microbioreactor under segmented flow. Cell Physiol Biochem 38:1883-1896

Sun T, Feng L, Gao X, Jiang L (2005) Bioinspired surfaces with special wettability. Acc Chem Res 38:644-652. doi:10.1021/ar040224c

Teo AJT et al (2017) Negative pressure induced droplet generation in a microfluidic flow-focusing device. Anal Chem 89:4387-4391. doi:10.1021/acs.analchem.6b05053

Tuvshindorj U, Yildirim A, Ozturk FE, Bayindir M (2014) Robust cassie state of wetting in transparent superhydrophobic coatings. ACS Appl Mater Interfaces 6:9680-9688. doi:10.1021/am502117a

Volpatti LR, Yetisen AK (2014) Commercialization of microfluidic devices. Trends Biotechnol 32:347-350. doi:10.1016/j. tibtech.2014.04.010

Wagner P, Fürstner R, Barthlott W, Neinhuis C (2003) Quantitative assessment to the structural basis of water repellency in natural and technical surfaces. J Exp Bot 54:1295-1303

Wang J, Yi P, Deng Y, Peng L, Lai X, Ni J (2017) Recovery behavior of thermoplastic polymers in micro hot embossing process. J Mater Process Technol 243:205-216. doi:10.1016/j. jmatprotec.2016.12.024

Wenzel RN (1949) Surface roughness and contact angle. J Phys Colloid Chem 53:1466-1467. doi:10.1021/j150474a015

Wiedemeier S et al (2011) Encapsulation of Langerhans' islets: microtechnological developments for transplantation. Eng Life Sci 11:165-173. doi:10.1002/elsc.201000146

$\mathrm{Wu} \mathrm{Z}$ et al (2016) Rose petal mimic surface by $\mathrm{TiO}_{2}$ sol-gel process. In: 2016 IEEE international conference on manipulation, manufacturing and measurement on the nanoscale (3M-NANO), 18-22 July 2016, pp 221-224. doi:10.1109/3M-NANO.2016.7824970

Xi H-D et al (2017) Active droplet sorting in microfluidics: a review. Lab Chip 17:751-771. doi:10.1039/C6LC01435F

Xue C-H, Jia S-T, Zhang J, Ma J-Z (2010) Large-area fabrication of superhydrophobic surfaces for practical applications: an overview. Sci Technol Adv Mater 11:033002. doi:10.1088/1468-6996/11/3/033002

Zang E et al (2013) Real-time image processing for label-free enrichment of Actinobacteria cultivated in picolitre droplets. Lab Chip 13:3707-3713. doi:10.1039/c3lc50572c 